\title{
The longitudinal change in anthropometric measurements and the association with physical function decline in Japanese community-dwelling frail elderly
}

\author{
Sachiko Izawa ${ }^{1}$, Hiromi Enoki ${ }^{1}$, Yoshihisa Hirakawa ${ }^{1}$, Mitsunaga Iwata ${ }^{2}$, Jun Hasegawa ${ }^{1}$, Akihisa Iguchi $^{1}$ \\ and Masafumi Kuzuya* \\ ${ }^{1}$ Department of Geriatrics, Nagoya University Graduate School of Medicine, 65 Tsuruma-cho, Showa-ku, Nagoya 466-8550, Japan \\ ${ }^{2}$ Emergency Department, Nagoya Ekisaikai Hospital, 4-66 Shonen-cho, Nakagawa-ku, Nagoya 454-8502, Japan
}

(Received 3 December 2008 - Revised 4 June 2009 - Accepted 21 July 2009 - First published online 14 September 2009)

Although anthropometric parameters have been extensively studied regarding their relationship to physical function status, the association between these parameters and the activity of daily living (ADL) function remains controversial. We investigated whether BMI or mid-upper arm circumference $(\mathrm{AC})$ is an indication of variation in the physical functioning of the frail elderly. The present study was a prospective cohort analysis of 543 community-dwelling frail elderly. Data included the participants' demographic characteristics, basic ADL, comorbidity and anthropometric measurements at baseline and at 2-year follow-up. Logistic regression models were used to investigate the association between ADL status and anthropometric measurements during the study period. Among the 543 participants, 418 maintained or improved their ADL status, while 125 showed an ADL decline during the study period. Multivariate logistic regression analysis showed that BMI and AC levels or ADL status at baseline were not independent predictors of the loss of ADL function or the decline in these anthropometric measurements during the study period, respectively. However, the decline in BMI and AC levels and the loss of ADL function were associated with each other during the study period. There is an association between the negative changes in anthropometric measurements during the follow-up period and the decline in ADL function during a 2-year follow-up in community-dwelling frail elderly.

Activities of daily living: Declining anthropometric measurement: Declining activity of daily living score: Elderly

The decline in physical performance is directly linked to the negative consequences of the reduced health and quality of life of elderly people. A number of studies have demonstrated relationships between physical disability and nutritional status, including anthropometric measurements, in the elderly ${ }^{(1,2)}$. Among anthropometric parameters, weight and BMI have been extensively studied in terms of their relationship with physical function status. In cross-sectional analysis, community-dwelling older people with BMI $30 \mathrm{~kg} / \mathrm{m}^{2}$ or higher than $30 \mathrm{~kg} / \mathrm{m}^{2}$ were associated with a greater probability of functional limitation ${ }^{(3-6)}$. In contrast, severe disability in institutionalised elderly subjects was associated with low waist/hip ratio $^{(7)}$. Longitudinal studies have demonstrated that weight loss is associated with an increase in the risk of becoming disability compared with weight stability in community-dwelling elderly ${ }^{(8-11)}$. In contrast, it has been reported that obesity (BMI: $35 \mathrm{~kg} / \mathrm{m}^{2}$ or greater) and weight gain are risk factors for functional decline between 3 and 4 years later in mean aged 71 years participants ${ }^{(12)}$. Thus, the causal relationships between anthropometric measurements and physical functional ability remain controversial.

Most prior studies utilise body weight or BMI as an anthropometric parameter to examine the relationships between nutritional status and functional ability. We recently reported that the height and weight of older people with activity of daily living (ADL) ${ }^{(13,14)}$ impairment are not likely to be measured at home or to be regularly measured in the community $^{(15)}$. In fact, approximately one-third of disabled elderly living at home lack recent height or weight measurements ${ }^{(15)}$. We proposed that anthropometric measurements of the midarm may be a more practical and suitable index not only for nutritional assessment but also for capturing the vulnerable subset of older people living in the community ${ }^{(16)}$. However, no data were available concerning the relationships between mid-arm measurements and ADL status.

In the present study, we investigated whether anthropometric measurements such as the BMI and mid-arm circumference (AC) of frail elderly individuals are an accurate indication of variation in their physical functioning using a prospective cohort study of community-dwelling frail elderly.

\section{Methods \\ Subjects \\ The present study employed baseline data for the subgroup of participants in the Nagoya Longitudinal Study for Frail Elderly and data on the mortality and hospitalisation of these participants during the 2-year follow-up period. Details}


of the participants and the Nagoya Longitudinal Study for Frail Elderly have been published elsewhere ${ }^{(17,18)}$. The study population consisted of 952 community-dwelling frail elderly (men: 355, mean age 78.5 years (range: 65-101); women: 597, mean age 81.6 years (range: 65-102)) with physical or mental disability in some degree. They were eligible for long-term care insurance ${ }^{(19,20)}$, lived in Nagoya City, and were provided visiting nurse services by the Nagoya City Health Care Service Foundation for Older People, which has seventeen visiting nursing stations associated with care-managing centres. Eligibility of long-term care insurance is strictly a matter of age, physical and mental status, and whether or not the individual has undergone medical procedures. These Nagoya Longitudinal Study for Frail Elderly participants, who were enrolled between 1 December 2003 and 31 January 2004, were scheduled to undergo comprehensive in-home assessments by trained nurses at the baseline, and at 6,12 and 24 months. At 3-month intervals, data were collected about any important events in the lives of the participants, including admission to the hospital, long-term care facilities placement and mortality. In the present study, a fall was defined as a sudden, unexpected descent from a standing, sitting or horizontal position, and slipping from a chair or wheelchair. Participants found down on the floor were also included in falls. After 1 year, the participants or family member caregivers were asked the fall experience by trained nurses. Written informed consent for participation, according to procedures approved by the institutional review board of Nagoya University Graduate School of Medicine, was obtained from the patients or, for those with physiciandiagnosed substantial cognitive impairment, from a surrogate (usually the closest relative or legal guardian), and from family member caregivers.

\section{Data collection}

The data were collected at the clients' homes using structured interviews with patients or surrogates and caregivers, and from care-managing centre records taken by trained nurses. The data included clients' demographic characteristics, depressive symptoms as assessed by the short version of the Geriatric Depression Scale ${ }^{(21)}$, and ADL was assessed by the 20-point Barthel Index with some modification (feeding, mobility on bed, bathing, grooming, dressing, using the toilet, walking inside and outside, transferring and using stairs) using summary scores ranging from 0 (total disability) to 20 (no disability). For each ADL task, nurses rated participants as independent (a score of 2, able to perform the activity without help), partially dependent (a score of 1, requiring some assistance) or completely dependent (a score of 0 , needing help for the entire activity). Nurse ratings were based on direct observation, questioning of patients and speaking with family members and caregivers. Information obtained from care-managing centre records included data on the following physician-diagnosed chronic conditions: IHD; congestive heart failure; cerebrovascular disease; diabetes mellitus; dementia; cancer; neurodegenerative disorders including Parkinson's disease; other diseases comprising the Charlson comorbidity index ${ }^{(22)}$, which represents the sum of weighted indices taking into account the number and seriousness of pre-existing comorbid conditions.

\section{Anthropometry}

Height and weight data were generally measured at home and collected by trained nurses using the methods described by the World Health Organization ${ }^{(23)}$. The visiting nurses were asked to measure the height or weight of participants at home as much as possible. If body weight measurements could not be taken at home for some reason, recorded or self-reported weight data obtained sometime within the last month were used. If the participants have some cognitive impairment, weight data were obtained form a surrogate or family member caregivers. Weight was measured in light clothing without shoes using a portable weight scale at home. Height was generally measured in an upright position using a tape measure attached to the wall. However, when participants could not maintain an upright position, height measurements were obtained in a prone position. Although there are surrogate methods for obtaining height using estimation equations based on body segment lengths, these methods have not been validated in the Japanese elderly. Height measurements were unavailable for subjects ( $n 342,35.9 \%$ ) with severe kyphosis (defined as any subject whose kyphosis made it impossible for the visiting nurse to make a convenient or reliable height measurement) or severe muscle and arterial contracture.

Measurement ${ }^{(23)}$ of the triceps skin fold (TSF) thickness (to the nearest $0.1 \mathrm{~mm}$ ) was made using skinfold callipers and $\mathrm{AC}$ (to the nearest $0 \cdot 1 \mathrm{~cm}$ ) using a flexible measuring tape on the right side of the participant's body, unless affected by disability or disease. Arm area (AMA) was calculated using the standard formula ${ }^{(24)}: \mathrm{AMA}=(\mathrm{AC}(\mathrm{cm})-0.3142 \times \mathrm{TSF}$ $(\mathrm{mm}))^{2} / 4 \pi$. These measurements were taken at least twice by each trained nurse according to the instruction sheet, and the reported values were the means of the repeated measurements.

Among the 952 participants, $207(21.7 \%)$ subjects died, 41 (4.3\%) were admitted to hospitals, $78(8.2 \%)$ had long-term care placement and $83(8.7 \%)$ declined to participate at some point during the 2-year follow-up.

Therefore, the ADL levels of a total of 543 participants (men: 201; women: 342) were assessed after the 2-year period and were used for the analysis in the present study. The subjects who died during the 2-year follow-up were older (mean age: 82.1 years $v .79 .8$ years, $P=0.001$ ), and mean scores of BMI, AC, TSF, AMA and basic ADL at baseline were significantly lower than those of 543 participants analysed in the present study (BMI: 18.8 v. 21.2, $\mathrm{p}<0.001$; AC: 21.8 v. 24.0, $P<0.001$; TSF: 1.3 v. 1.6, $P<0.001$; AMA: 26.6 v. $30.3, P<0.001$; basic ADL: 7.7 v. 10.7, $P<0.001)$. The 50th percentile of ADL scores at baseline (range: 0-20) was 12. There were seventy-five participants who had an ADL score of 0 at baseline, and 291 participants who had ADL scores of 12 or more at baseline among all participants ( $n$ 543). Out of 543 participants, $280(51.6 \%)$ and $471(86.7 \%)$ were available for their BMI and mid-arm measurements at both baseline and at 2 years, respectively.

\section{Statistical analysis}

The Student's $t$ test and $\chi^{2}$ test were used to compare differences between participants with ADL decline and those without decline (improved or stable ADL function). 
The main dependent variable was a change in the ability to perform ADL tasks over the 2-year follow-up period. We distinguished two levels of categorical change: (i) participants with no change or improved ADL score at the 2-year endpoint compared with baseline; (ii) participants with a decline in ADL score from baseline to the 2-year endpoint.

Univariate and multivariate logistic regression models were used to assess the following two questions: (1) Do the anthropometric measurements (BMI or AC) at baseline predict ADL status change during the 2-year follow-up? (2) Does baseline ADL status predict the loss of these anthropometric parameters during the study period? To avoid the floor effect of ADL score, the analysis was also conducted with participants with ADL scores of 12 (the 50th percentile of basic ADL at baseline) or higher at baseline. The following baseline data were used in univariate analysis: the sex; age; ADL score; Charlson comorbidity index; living arrangement; the presence or absence of chronic diseases; BMI; AC; TSF; AMA; Geriatric Depression Scale score; the fall and hospitalisation experience during the 2-year period. The covariates included in the multivariate analysis were variables associated with dependent variables with $P<0.05$ in univariate analysis. The risk of a variable was expressed as an OR with a corresponding $95 \%$ CI.

We also examined the association between ADL score decline and the change in anthropometric measurements change during the study period using logistic regression analysis. The ADL score changes were categorised into three groups: improved/stable; 1 point change; $\geq 2$ points change. The changes in $\mathrm{BMI}$ or $\mathrm{AC}$ were categorised into three or four groups: BMI, increase $/$ stable, $<1.0$ and $\geq 1.0 \mathrm{~kg} / \mathrm{m}^{2}$; $\mathrm{AC}$, increase/stable, $\leq 0.5,0.6-1.5$ and $\geq 1.6 \mathrm{~cm}$. Again the covariates included in the multivariate analysis were variables associated with dependent variables in univariate analysis.

To evaluate the relationship between the decline in BMI and $\mathrm{AC}$ levels and the decline in ADL scores during the study period, Spearman's rank correlation coefficient was used. Partial rank correlation coefficients adjusted for age and sex were also used to measure the relationships between the decline in BMI and AC levels and the decline in ADL scores during the study period.

All analyses were performed using the Statistical Package for the Social Sciences (SPSS, Inc., Chicago, IL, USA) Version 15.0. A probability value of 0.05 or less was considered significant.

\section{Results}

At the baseline, total dependent participants (basic ADL score of 0$)$ were $143(15.0 \%)$ and total independent participants (basic ADL score of 20) were 61 (6.4\%), among the 952 participants. Among the 543 participants, 418 (77.0\%) participants maintained (n 390) or improved $(n$ 28) their ADL status, while $125(23 \%)$ showed an ADL decline during the study period.

Table 1 shows the comparisons of baseline characteristics of participants with or without ADL decline during the 2 -year period. No differences were observed in baseline BMI, AC, TSF and AMA between participants in the two groups. A higher prevalence of hypertension and neurodegenerative disease and a higher ADL score at baseline were observed in participants with ADL decline. The prevalence rates of hospitalisation and falls during the 2-year period were significantly higher for those with ADL decline

Table 1. Baseline characteristics of the 543 care recipients (Mean values and standard deviations)

\begin{tabular}{|c|c|c|c|c|c|}
\hline & \multicolumn{5}{|c|}{ Change in basic ADL scores during 2-year follow-up } \\
\hline & \multicolumn{2}{|c|}{ Improved/stable ( $n$ 418) } & \multicolumn{2}{|c|}{ Declined $(n$ 125) } & \multirow[b]{2}{*}{$P$} \\
\hline & Mean & SD & Mean & SD & \\
\hline Men/women, $n(\%$ of men/total) & $150 / 268(35.9)$ & & $51 / 74(40 \cdot 8)$ & & 0.318 \\
\hline Age $\left(\right.$ years) ${ }^{\star}$ & 79.6 & $7 \cdot 8$ & $80 \cdot 5$ & 9.5 & 0.285 \\
\hline BMI $\left(\mathrm{kg} / \mathrm{m}^{2}\right)^{\star}$ & $21 \cdot 2$ & $4 \cdot 0$ & $21 \cdot 2$ & 3.6 & 0.973 \\
\hline Mid-arm circumference $(\mathrm{cm})^{\star}$ & $24 \cdot 0$ & $4 \cdot 3$ & $24 \cdot 0$ & $4 \cdot 1$ & 0.854 \\
\hline Triceps skin fold $(\mathrm{cm})^{\star}$ & 1.6 & 0.9 & 1.5 & 0.9 & 0.168 \\
\hline Arm muscle area $\left(\mathrm{cm}^{2}\right)^{\star}$ & $30 \cdot 0$ & $11 \cdot 8$ & $31 \cdot 0$ & $12 \cdot 2$ & 0.399 \\
\hline Basic ADL (range $0-20)^{*}$ & $10 \cdot 1$ & $7 \cdot 2$ & $12 \cdot 8$ & 4.7 & $<0.001$ \\
\hline GDS-15 (range, $0-15)^{*}$ & $6 \cdot 7$ & 3.6 & $7 \cdot 3$ & $3 \cdot 6$ & 0.138 \\
\hline Charlson comorbidity index ${ }^{*}$ & $2 \cdot 2$ & 1.6 & $2 \cdot 1$ & 1.6 & 0.602 \\
\hline Living alone (\% of total) & $19 \cdot 1$ & & $16 \cdot 9$ & & 0.590 \\
\hline \multicolumn{6}{|l|}{ Chronic diseases (\% of total) $\dagger$} \\
\hline IHD & $11 \cdot 6$ & & $10 \cdot 5$ & & 0.757 \\
\hline Congestive heart failure & $11 \cdot 0$ & & $10 \cdot 5$ & & 0.884 \\
\hline Cerebrovascular disease & $43 \cdot 5$ & & $43 \cdot 9$ & & 0.947 \\
\hline Diabetes mellitus & 11.9 & & $13 \cdot 2$ & & 0.713 \\
\hline Dementia & $33 \cdot 3$ & & $31 \cdot 6$ & & 0.729 \\
\hline Cancer & $6 \cdot 8$ & & $9 \cdot 6$ & & 0.311 \\
\hline Hypertension & $23 \cdot 2$ & & $32 \cdot 8$ & & 0.031 \\
\hline Pressure sore & $11 \cdot 7$ & & $6 \cdot 4$ & & 0.089 \\
\hline Neurodegenerative disorders & $9 \cdot 8$ & & $19 \cdot 2$ & & 0.005 \\
\hline
\end{tabular}

ADL, activity of daily living; GDS-15, geriatric depression scale.

* Student's $t$ test, others were analysed by $\chi^{2}$ test (changeless improvement $v$. decline).

† Chronic diseases: physician-diagnosed chronic conditions. 
(40.0\%, $P<0.012$ and $46.6 \%, P<0.002$, respectively) than for those who were stable or showed improvement in ADL ( 28.2 and $31.3 \%$, respectively).

Table 2 shows the average changes in ADL scores and the average changes in the BMI and AC levels of participants with or without ADL score decline during the 2-year period. Significant decreases in BMI and AC during the 2-year period were observed in participants with loss of ADL function compared with those with a stable or improved ADL score, although there was no difference in TSF or AMA change during the 2-year period between two groups (data not shown).

To examine whether the anthropometric measurements (BMI or AC) at baseline may predict ADL status change during the 2-year follow-up, logistic regression analysis was conducted. As shown in Table 3, BMI or AC levels at baseline were not an independent predictor of the loss of ADL function during the study period not only in univariate analysis but also in multivariate analysis. When the analysis was conducted for participants with ADL scores of 12 or higher at baseline, again there was no association between the decline in ADL status and $\mathrm{BMI}$ and $\mathrm{AC}$ at baseline.

During the 2-year period, 94 among 280 participants (33.6\%) and 165 among 471 participants $(35.0 \%)$ experienced a decline in their BMI or AC levels, respectively. The multivariate logistic analysis demonstrated that baseline ADL status was not associated with the loss of these anthropometric parameters during the study period (BMI: OR, 1.02; $95 \% \mathrm{CI}$, 0.97, 1.08 and AC: OR, 0.981; $95 \%$ CI, 0.95, 1.01).

There were correlations between the levels of decline in BMI and $\mathrm{AC}$ and changes in ADL score during the study period in all participants, as well as those excluded for having an ADL score 0 or with an ADL score of 12 or higher at baseline. These correlations persisted after adjusting for the age and sex of the participants (Table 4).

We next examined the association between the decline in ADL performance and BMI or AC change during the study period using logistic regression analysis (Table 5). Univariate as well as multivariate analysis showed that a larger decline in BMI $\left(\geq 1.0 \mathrm{~kg} / \mathrm{m}^{2}\right)$ and the highest level of AC decline $(\geq 1.6 \mathrm{~cm})$ compared with stable or increasing BMI and

Table 2. Change in anthropometric measurements and basic activity of daily living (ADL) scores during the 2-year period

(Mean values and standard deviations)

\begin{tabular}{|c|c|c|c|c|c|}
\hline & \multicolumn{4}{|c|}{ A change in basic ADL scores } & \multirow[b]{3}{*}{$P$} \\
\hline & \multicolumn{2}{|c|}{$\begin{array}{c}\text { Improved/ } \\
\text { stable }(n 418)\end{array}$} & \multicolumn{2}{|c|}{$\begin{array}{l}\text { Declined } \\
(n \text { 125) }\end{array}$} & \\
\hline & Mean & SD & Mean & SD & \\
\hline \multicolumn{6}{|c|}{ Basic ADL score (range $0-20)^{\star}$} \\
\hline Change during 2 years & 0.1 & 0.5 & -3.5 & 3.43 & $<0.001$ \\
\hline \multicolumn{6}{|l|}{ BMI $\left(\mathrm{kg} / \mathrm{m}^{2}\right)^{*}$} \\
\hline Baseline & $21 \cdot 2$ & 4.0 & $21 \cdot 3$ & 3.5 & 0.788 \\
\hline After the 2-year period & $21 \cdot 2$ & $4 \cdot 1$ & $20 \cdot 7$ & 3.5 & 0.399 \\
\hline Change during 2 years & -0.005 & $1 \cdot 2$ & -0.60 & $1 \cdot 8$ & 0.010 \\
\hline \multicolumn{6}{|c|}{ Mid-arm circumference $(\mathrm{cm})^{\star}$} \\
\hline Baseline & $24 \cdot 1$ & $4 \cdot 2$ & $24 \cdot 1$ & 4.0 & 0.916 \\
\hline After the 2-year period & 23.6 & 4.5 & $23 \cdot 1$ & 3.9 & 0.327 \\
\hline Change during 2 years & -0.3 & 1.9 & $-1 \cdot 0$ & $2 \cdot 7$ & 0.015 \\
\hline
\end{tabular}

* Student's $t$ test.
AC measurements were associated with the loss of ADL performance during the 2-year period. Conversely, a decline in $\mathrm{ADL}$ score during the 2-year period of $\geq 2$ points was associated with a loss of BMI or AC scores in univariate analysis. These associations persisted after adjustments for potential confounders were made in multivariate models (Table 5). When multivariate analysis was conducted among participants with an ADL score of 12 or higher, similar associations were detected between the decline in BMI and AC levels and the loss of ADL function (model 2 in Table 5).

\section{Discussion}

The present study examined the association between anthropometric measurements at baseline or longitudinal changes in those measurements and the degree of disability or longitudinal physical function decline during a 2-year follow-up in community-dwelling frail Japanese elderly. Although, as far as we know, there has been no report demonstrating that $\mathrm{AC}$ acts as a predictor of physical functional impairment, a previous report has demonstrated that greater BMI (BMI: $35 \mathrm{~kg}$ / $\mathrm{m}^{2}$ or greater) at baseline was associated with physical function decline in community-dwelling frail elderly ${ }^{(12)}$. In contrast, in the present study, we clearly showed that baseline BMI or AC was not a predictor for the decline in ADL performance. These differences may be due to the different ethnicity of the participants or the presence of participants with lower BMI (mean BMI: $21.2 \mathrm{~kg} / \mathrm{m}^{2}$ ) and lower physical function in the present study compared with those in previous studies. However, it showed be noted that the means of BMI and $\mathrm{AC}$ levels at baseline in the participants of the present cohort study were similar to those of the standard Japanese older population as previously reported ${ }^{(16,25)}$. It is possible that poor nutritional status, which reflects anthropometric parameters, contributes to the development of functional disability; likewise, it is also plausible that disability at baseline may lead to lifestyle changes, which in turn result in the decline in anthropometric parameters. However, we also demonstrated that lower ADL function at baseline was not a risk for the loss of BMI or AC levels during the follow-up period. These results suggested that there are neither causal relationships between basal lower anthropometric

Table 3. Logistic regression analysis to identify independent predictors of declining basic activity of daily living (ADL) score (OR values with $95 \% \mathrm{Cl}$ )

\begin{tabular}{lccc}
\hline & OR & $95 \% \mathrm{Cl}$ & $P$ \\
\hline BMI $\left(\mathrm{kg} / \mathrm{m}^{2}\right)$ & & & \\
$\quad$ Unadjusted & 1.00 & $0.94,1.07$ & 0.972 \\
Adjusted* & 0.98 & $0.91,1.06$ & 0.625 \\
Adjusted $\dagger$ & 0.97 & $0.89,1.06$ & 0.558 \\
Mid-arm circumference $(\mathrm{cm})$ & 1.00 & $0.95,1.04$ & 0.853 \\
$\quad$ Unadjusted & 0.98 & $0.93,1.04$ & 0.467 \\
Adjusted* & 0.96 & $0.90,1.04$ & 0.327 \\
Adjusted $\dagger$ & &
\end{tabular}

The 50th percentile of basic ADL scores at baseline was 12 in the present study.

*Adjusted includes sex, age, presence or absence of hypertension and neurodegenerative disease, hospitalisation and fall experience during the 2-year study period and the score of basic $A D L$ at baseline.

† Analysis was conducted for participants with basic ADL score of 12 or higher at baseline. 
Table 4. Correlations between changes in basic activity of daily living (ADL) score during 2-year study period and anthropometric measurements during 2-year study period

\begin{tabular}{|c|c|c|c|}
\hline \multirow[b]{2}{*}{$\begin{array}{l}\text { Changes in levels } \\
\text { during 2-year period }\end{array}$} & \multicolumn{3}{|c|}{ Change of $A D L$ score during 2-year follow-up } \\
\hline & $\begin{array}{l}\text { All participants } \\
(n 543)\end{array}$ & $\begin{array}{l}\text { Excluded basic } \\
\text { ADL score of } 0 \\
\text { at baseline } \\
(n 468)\end{array}$ & $\begin{array}{c}\text { Basic ADL } \\
\text { score of } 12 \\
\text { or higher† } \\
\text { at baseline } \\
\quad(n 291)\end{array}$ \\
\hline \multicolumn{4}{|l|}{$\mathrm{BMI}\left(\mathrm{kg} / \mathrm{m}^{2}\right)$} \\
\hline Crude & $0.123^{*}$ & 0.117 & 0.134 \\
\hline Adjusted $\ddagger$ & $0.149^{*}$ & $0.137^{*}$ & $0.191^{\star *}$ \\
\hline \multicolumn{4}{|c|}{ Mid-arm circumference $(\mathrm{cm})$} \\
\hline Crude & $0.157^{\star *}$ & $0 \cdot 169^{* *}$ & 0.099 \\
\hline Adjusted $\ddagger$ & $0.152^{\star \star}$ & $0.158^{\star *}$ & $0.169^{\star \star}$ \\
\hline
\end{tabular}

Mean values were significantly different: ${ }^{\star} P<0.05,{ }^{\star \star} P<0.01$.

†The 50th percentile of basic ADL scores at baseline was 12 in the present study. $\ddagger$ Adjusted for age and sex.

measurements and ADL function decline nor between basal poor ADL performance and a decrease in anthropometric parameters.

Previous prospective cohort studies emphasised that weight loss is a risk factor for the functional decline ${ }^{(8-12)}$. However, no studies allow the evaluation of causal relationships between weight loss and the functional decline. In the present study, we showed that physical functional change was well correlated with change in anthropometric parameters. In addition, logistic regression models demonstrated that the decline in circumference

(OR values with $95 \% \mathrm{Cl}$ ) anthropometric parameters was a predictor of the decline in ADL performance, and that, conversely, the decline in ADL performance was also a predictor of anthropometrics decline. These results may indicate that these factors, anthropometric parameters and ADL status, influence each other and decline simultaneously. BMI and AC are parameters of nutritional status in older adults ${ }^{(26,27)}$. Therefore, the results may indicate that nutritional status and ADL performance were well correlated and changed simultaneously through causal and consequential relationships. Theoretically, inadequate intake of nutrients, one of the manifestations of undernutrition, which can lead to loss of muscle protein as well as body mass, may explain the association between weight loss and decline in physical function. In contrast, physical functional decline may be a cause of weight loss through difficulties in eating, provisioning and cooking, which can be reflected by a decline in the food-related items of the ADL.

It is possible that there are third factors that might produce the association between the loss of anthropometric parameters and the decline in ADL performance during the follow-up period. The occurrence of new diseases or poor control of chronic disease during the follow-up period might be a candidate for the third factor. However, the association persisted even after adjusting for hospitalisation for acute illness during the study period, which suggests that poor health outcomes leading to hospitalisation did not contribute to these relationships.

The present study has several limitations. The results of the present study cannot be transferred to non-frail-independent

Table 5. Logistic regression analysis to identify independent predictors of declining basic activity of daily living (ADL) Score, declining BMI and arm

\begin{tabular}{|c|c|c|c|c|c|c|c|c|c|}
\hline & & & & \multicolumn{6}{|c|}{ Multivariate } \\
\hline & \multicolumn{3}{|c|}{ Unadjusted } & \multicolumn{3}{|c|}{ Model 1} & \multicolumn{3}{|c|}{ Model 2} \\
\hline & OR & $95 \% \mathrm{Cl}$ & $P$ & OR & $95 \% \mathrm{Cl}$ & $P$ & OR & $95 \% \mathrm{Cl}$ & $P$ \\
\hline \multicolumn{10}{|c|}{ Basic ADL decline v. improve/stable* } \\
\hline \multicolumn{10}{|c|}{ Change in BMI levels during 2-year period } \\
\hline Increase or stable & 1.00 & Reference & & 1.00 & Reference & & 1.00 & Reference & \\
\hline Decline $\left(<1.0 \mathrm{~kg} / \mathrm{m}^{2}\right)$ & 1.09 & $0.50,2.39$ & 0.833 & 0.90 & $0.31,2.09$ & 0.810 & 0.89 & $0.30,2.58$ & 0.822 \\
\hline Decline $\left(\geq 1.0 \mathrm{~kg} / \mathrm{m}^{2}\right)$ & 3.86 & $1.96,7.59$ & $<0.001$ & 3.64 & $1.73,7.65$ & 0.001 & 4.69 & $1.89,11.67$ & 0.001 \\
\hline \multicolumn{10}{|c|}{ Change in $A C$ levels during 2-year period } \\
\hline Increase or stable & 1.00 & Reference & & 1.00 & Reference & & 1.00 & Reference & \\
\hline Decline $(\leq 0.5 \mathrm{~cm})$ & 1.55 & $0.66,3.65$ & 0.319 & 1.52 & $0.60,3.83$ & 0.373 & 1.00 & $0.31,3.25$ & 0.998 \\
\hline Decline $(0.6-1.5 \mathrm{~cm})$ & 1.77 & $0.91,3.45$ & 0.092 & $2 \cdot 54$ & $1 \cdot 20,5 \cdot 38$ & 0.015 & $2 \cdot 80$ & $1.02,7.69$ & 0.045 \\
\hline Decline $(\geq 1.6 \mathrm{~cm})$ & 2.75 & $1.63,4.64$ & $<0.001$ & 3.45 & $1.88,6.35$ & $<0.001$ & $3 \cdot 18$ & $1.40,7.20$ & 0.006 \\
\hline \multicolumn{10}{|c|}{ Loss of BMI levels $v$. increase/stable $\dagger$} \\
\hline \multicolumn{10}{|c|}{ Change in basic ADL score during 2-year period } \\
\hline Improved or stable & 1.00 & Reference & & 1.00 & Reference & & 1.00 & Reference & \\
\hline Decline (1 point) & 1.95 & $0.87,4.42$ & 0.107 & 1.94 & $0.77,4.84$ & 0.158 & $2 \cdot 67$ & $0.93,7.70$ & 0.069 \\
\hline Decline ( $\geq 2$ points) & 2.33 & $1.20,4.55$ & 0.013 & 3.28 & $1.50,7 \cdot 17$ & 0.003 & $3 \cdot 28$ & $1 \cdot 32,8 \cdot 16$ & 0.011 \\
\hline \multicolumn{10}{|c|}{ Loss of AC levels $v$. increase/stable } \\
\hline \multicolumn{10}{|c|}{ Change in basic ADL score during 2-year period } \\
\hline Improved or stable & 1.00 & Reference & & 1.00 & Reference & & 1.00 & Reference & \\
\hline Decline (1 point) & 1.40 & $0.74,2.66$ & 0.307 & 1.17 & $0.55,2.49$ & 0.678 & 1.01 & $0.40,2.58$ & 0.981 \\
\hline Decline ( $>2$ points) & 2.90 & $1.72,4.89$ & $<0.001$ & 3.62 & $1.95,6.73$ & $<0.001$ & 2.94 & $1.35,6.38$ & 0.007 \\
\hline
\end{tabular}

The 50th percentile of basic ADL scores at baseline was 12 in the present study.

* Multivariate analysis includes sex, age, presence or absence of hypertension and neurodegenerative disease, hospitalisation and fall experience during the 2-year period and the score of basic ADL at baseline. BMI: $n$ 135. mid-arm circumference (AC): $n 416$. Model 2 participants that had a basic ADL score of 12 or higher at baseline. BMI: $n 182$. AC: $n 245$.

† Multivariate analysis includes sex, age, living alone, regular medical checkups, cerebrovascular disease, hospitalisation during the 2-year period and the score of BMI at baseline. Participants had a basic ADL score of 2 or higher at baseline ( $n$ 225). Model 2 participants that had a basic ADL score of 12 or higher at baseline. $n 172$.

$\ddagger$ Multivariate analysis includes sex, age, living alone, regular medical checkups, cerebrovascular disease, hospitalisation during the 2-year period and the score of AC at baseline. Participants had a basic ADL score of 2 or higher at baseline. $n$ 342. Model 2 participants that had a basic ADL score of 12 or higher at baseline ( $n$ 228). 
older individuals, since there are many differences between the participants in Nagoya Longitudinal Study for Frail Elderly and standard non-frail older people, including differences in ADL levels and comorbidity. There was a possibility that the presence of lower BMI levels and fewer obese individuals in our cohort may have affected the present results. In addition, these findings may not be generalisable to other populations, given that they may have been influenced by cultural differences, health practices and a variety of social and economic factors. The mechanisms underlying the association between the decline in BMI/AC levels and declining ADL score during the 2-year follow-up period are unclear in the present study. Future study is needed to examine whether the ADL scores and anthropometric measurements of these frail elderly with functional limitations in the community decrease concurrently.

The present study showed that anthropometric measurements at baseline were not a predictor of physical function decline during a 2-year follow-up in community-dwelling elderly. An association was found between negative changes in anthropometric measurements during the follow-up period and the decline in ADL function.

\section{Acknowledgements}

The authors wish to thank all the patients, caregivers and the many nurses participating in the study, and the Nagoya City Health Care Service Foundation for Older People for their vigorous cooperation. The present study was supported by a Grant-in-Aid for the Comprehensive Research on Aging and Health from the Ministry of Health, Labor and Welfare of Japan, and a grant from Mitsui Sumitomo Insurance Welfare Foundation. The authors have no conflicts of interest with the manufactures of any drug evaluated in the present paper. S. I. contributed to the analysis of data and the writing of the manuscript. H. E. contributed to the analysis and interpretation data. Y. H. contributed to conduct of study and interpretation of data. M. I. contributed to analysis and interpretation of data. J. H. contributed to acquisition of data. A. I. contributed to the providing of significant advice. M. K. contributed to the study concept and design, the interpretation of data, and was performing quality control of the present study.

\section{References}

1. Stuck AE, Walthert JM, Nikolaus T, et al. (1999) Risk factors for functional status decline in community-living elderly people: a systematic literature review. Soc Sci Med 48, 445-469.

2. Topinková E (2008) Aging, disability and frailty. Ann Nutr Metab 52, Suppl. 1, 6-11.

3. Zoico E, Di Francesco V, Guralink JM, et al. (2004) Physical disability and muscular strength in relation to obesity and different body composition indexes in a sample of healthy elderly women. Int J Obes Relat Metab Disord 28, 234-241.

4. Larrieu S, Pérès K, Letenneur L, et al. (2004) Relationship between body mass index and different domains of disability in older persons: the 3C study. Int J Obes Relat Metab Disord 28, 1555-1560.

5. Friedmann JM, Elasy T \& Jensen GL (2001) The relationship between body mass index and self-reported functional limitation among older adults: a gender difference. J Am Geriatr Soc 49 $398-403$.
6. Chen H \& Guo X (2008) Obesity and functional disability in elderly Americans. J Am Geriatr Soc 56, 689-694.

7. Romagnoni F, Zuliani G, Bollini C, et al. (1999) Disability is associated with malnutrition in institutionalized elderly people. The I.R.A. Study. Istituto di Riposo per Anziani. Aging (Milano) 11, 194-199.

8. Launer LJ, Harris T, Rumpel C, et al. (1994) Body mass index, weight change, and risk of mobility disability in middle-aged and older women. The epidemiologic follow-up study of NHANES I. JAMA 271, 1093-1098.

9. Tully CL \& Snowdon DA (1995) Weight change and physical function in older women: findings from the Nun Study. $J$ Am Geriatr Soc 43, 1394-1397.

10. Al Snih S, Raji MA, Markides KS, et al. (2005) Weight change and lower body disability in older Mexican Americans. J Am Geriatr Soc 53, 1730-1737.

11. Ritchie CS, Locher JL, Roth DL, et al. (2008) Unintentional weight loss predicts decline in activities of daily living function and life-space mobility over 4 years among communitydwelling older adults. J Gerontol A Biol Sci Med Sci 63, 67-75.

12. Jensen GL \& Friedmann JM (2002) Obesity is associated with functional decline in community-dwelling rural older persons. $J$ Am Geriatr Soc 50, 918-923.

13. Mahoney F \& Barthel DW (1965) Functional evaluation: The Barthel Index. Md State Med J 14, 61-65.

14. Collin C, Wade DT, Davies S, et al. (1998) The Barthel ADL Index: a reliability study. Int Disabil Stud 10, 61-63.

15. Izawa S, Enoki H, Hirakawa Y, et al. (2007) Lack of body weight measurement is associated with mortality and hospitalization in community-dwelling frail elderly. Clin Nutr 26, 764-770.

16. Enoki H, Kuzuya M, Masuda Y, et al. (2007) Anthropometric measurements of mid-upper arm as a mortality predictor for community-dwelling Japanese elderly: the Nagoya Longitudinal Study of Frail Elderly (NLS-FE). Clin Nutr 26, 597-604.

17. Kuzuya M, Masuda Y, Hirakawa Y, et al. (2006) Underuse of medications for chronic diseases in the oldest of communitydwelling older frail Japanese. J Am Geriatr Soc 54, 598-605.

18. Kuzuya M, Masuda Y, Hirakawa Y, et al. (2006) Day care service use is associated with lower mortality in communitydwelling frail older people. J Am Geriatr Soc 9, 1364-1371.

19. Campbell JC \& Ikegami N (2000) Long-term care insurance comes to Japan. Health Aff (Millwood) 19, 26-39.

20. Tsutsui T \& Muramatsu N (2005) Care-needs cerfication in the long-term care insurance system of Japan. J Am Geriatr Soc 53, 522-527.

21. Arai Y, Kudo K, Hosokawa T, et al. (1997) Reliability and validity of the Japanese version of the Zarit Caregiver Burden interview. Psychiatry Clin Neurosci 51, 281-287.

22. Charlson ME, Pompei P, Ales KL, et al. (1987) A new method of classifying prognostic comorbidity in longitudinal studies: development and validation. J Chronic Dis 40, 373-383.

23. World Health Organization (1986) Use and interpretation of anthropometric indicators of nutritional status: report of a WHO working group. Bull World Health Organ 64, 929-941.

24. Heymsfield SB, McManus C, Smith J, et al. (1982) Anthropometric measurement of muscle mass: revised equations for calculating bone-free arm muscle area. Am J Clin Nutr 36, 680-690.

25. Japanese Anthropometric Reference Data (2002) JARD 2001, Japanese Anthropometric Reference Data (in Japanese). Jpn J Nutr Assess 19, Suppl., 45-81.

26. Gariballa S \& Forster S (2007) Malnutrition is an independent predictor of 1-year mortality following acute illness. Br J Nutr 98, 332-336.

27. Izawa S, Kuzuya M, Okada K, et al. (2006) The nutritional status of frail elderly with care needs according to the mininutritional assessment. Clin Nutr 25, 962-967. 